\title{
Primary Pulmonary Lymphoepithelioma-Like
}

\section{Carcinoma Response Favorably To Nivolumab: A Case Report}

This article was published in the following Dove Press journal: OncoTargets and Therapy

\author{
Zhi-Xin Qiu' \\ Ping Zhou ${ }^{2}$ \\ Ke Wang' \\ 'Department of Respiratory and Critical \\ Care Medicine, West China Hospital, \\ Sichuan University, Chengdu, People's \\ Republic of China; ${ }^{2}$ Department of \\ Pathology, West China Hospital, Sichuan \\ University, Chengdu, People's Republic of \\ China
}

Objectives: Lymphoepithelioma-like carcinoma (LELC) is a rare subtype of non-small cell lung cancer (NSCLC). No clinical trials have been performed, and no course of treatment for LELC has been established because of it's rarity. This study presents a patient with primary pulmonary LELC, who was treated with nivolumab, and responded favorably.

Materials and methods: A patient with primary pulmonary LELC was treated using nivolumab.

Result: The patient responded well to immunotherapy with nivolumab. After five cycles of the nivolumab, the size of the tumor and the lesions of the liver became smaller. A blood test showed that CYFRA21-1 and NSE had dramatically decreased from before, especially the CYFRA21-1.

Conclusion: EBV-positive pulmonary LELC with high expression of PD-L1 may derive a benefit from PD-1/PD-L1 blockade.

Keywords: LELC, NSCLC, nivolumab, PD-L1

\section{Introduction}

Lymphoepithelioma-like carcinoma (LELC), which has similar morphology with undifferentiated nasopharyngeal carcinoma (NPC), is a rare subtype of non-small cell lung cancer (NSCLC), and has often been identified in young non-smokers. ${ }^{1}$ The incidence of LELC is $\sim 0.7 \%$ of all NSCLC and it was first described by Begin et $\mathrm{al}^{2}$ in 1987. LELC is usually associated with Epstein-Barr virus (EBV) infection, and has been documented with specific ethnic and regional preferences, especially patients from Asia, such as Guangdong Province, Hong Kong, and Taiwan in China. ${ }^{3-8}$

Owing to its rarity, no clinical trials have been performed, and no course of treatment for LELC has been established. Only case reports and retrospective series have been reported. Treatment at advanced stages has usually relied on multimodal therapy, including chemotherapy and radiotherapy. ${ }^{9}$ In recent years, significant advances have been made in cancer immunotherapy. Here, we present a patient with LELC of the lung, who was treated with nivolumab, responding favorably.

\section{Case Presentation}

A 56-year-old never smoking woman was admitted to our Lung Cancer Center with no symptoms, but a big mass was found in the left lower lobe (Computed tomography (CT) of the chest) through annual healthy check-up. She denied hepatitis,
Department of Respiratory and Critical

Care Medicine, West China Hospital,

Sichuan University, Chengdu 61004I,

People's Republic of China

Tel +86-28 86298115

Email wang2ke@I26.com 
tuberculosis, or other infectious disease, and had no history of smoking, drinking, allergies, or surgery. There was no obvious change in body weight.

An enhanced CT of the chest, whole abdomen, brain, and nasopharyngeal and a whole body bone scan were arranged. The enhanced CT of the chest showed a bulky soft tissue mass in the left lower lobe with lobulated, burr sign, and the largest cross-section size was about $6.4 \times 6.0 \mathrm{~cm}$. Furthermore, the left lung door and mediastinal lymph nodes showed enlargement. There were no trachea bronchial stenosis and no pericardial or pleural effusion (Figures 1A1, A2, B1, and B2). The enhanced $\mathrm{CT}$ of the whole abdomen showed a kind of circular low density shadow of $4.1 \times 3.6 \mathrm{~cm}$ in the liver (Figure $1 \mathrm{C}$ ). The brain and nasopharyngeal enhanced CT and the whole body bone scan were roughly normal except some focal ischemia, deputy nose, and hypertrophy of bilateral inferior turbinate (Figures 1D-F). Serum tumor markers indicated cell keratin fragments antigen 21-1 (CYFRA21-1) and neural specificity enolase (NSE) were higher than the normal values $(10.38 \mathrm{ng} / \mathrm{mL}$ vs $<3 \mathrm{ng} / \mathrm{mL}$ and $22.62 \mathrm{ng} /$ $\mathrm{mL}$ vs $<15 \mathrm{ng} / \mathrm{mL}$, respectively). Carcinoembryonic antigen (CEA), serum carbohydrate antigen 125, 19-9, 15-3, and 72-4 (CA125, CA19-9, CA15-3, and CA72-4) were all negative. Other abnormal blood test results including heat shock protein $90 \alpha$ (HSP90 $\alpha$ ) and erythrocyte sedimentation (ESR), especially EBV-EA-IgG was 158.14 $\mathrm{RU} / \mathrm{mL}$ which was almost eight times higher than normal value.
In order to define the diagnosis, fiber optic bronchoscope (FOB) and percutaneous lung biopsy were performed. All levels of the bronchial lumen were normal through the FOB, and the biopsy showed only chronic inflammation. However, percutaneous lung biopsy prompted lymphoepithelioma-like carcinoma combined with the results of hematoxylin-eosin (HE) and immunohistochemical (IHC) staining. IHC staining demonstrated PCK (+), P40 (+), CK5/6 (+), EBER1/2-ISH (+), CK7 (-), TTF-1 (-), and PD-L1 ( + , positive proportion about $80 \%$, antibody SP142, ZSGB-BIO Company) (Figure 2). The findings from next generation sequencing (NGS) were negative for mutations in EGFR, KRAS, and BRAF and negative for rearrangements in ALK and ROS1. Then we diagnosed left lower lobe lymphoepithelioma-like carcinoma invading the pericardium and descending aorta, with metastasis of mediastinal lymph nodes and liver (cT4N2M1b, stage IVA).

The first treatment regimen was paclitaxel $(190 \mathrm{mg})$ plus carboplatin $(550 \mathrm{mg})$. After two cycles, we evaluated the curative effect according to enhanced CT of the chest and whole abdomen, and we found the size of tumor became larger (Figures 3A1 and A2) and the lesions of liver became more broader than before (Figures 3B1-B4, evaluation result was SD (according to RECIST 1.1)). We evaluated again after four cycles of chemotherapy, and found the CT imaging demonstrated significant progression of disease (Figure 4, evaluation result was PD). After consulting with the patient and considering the high expression of

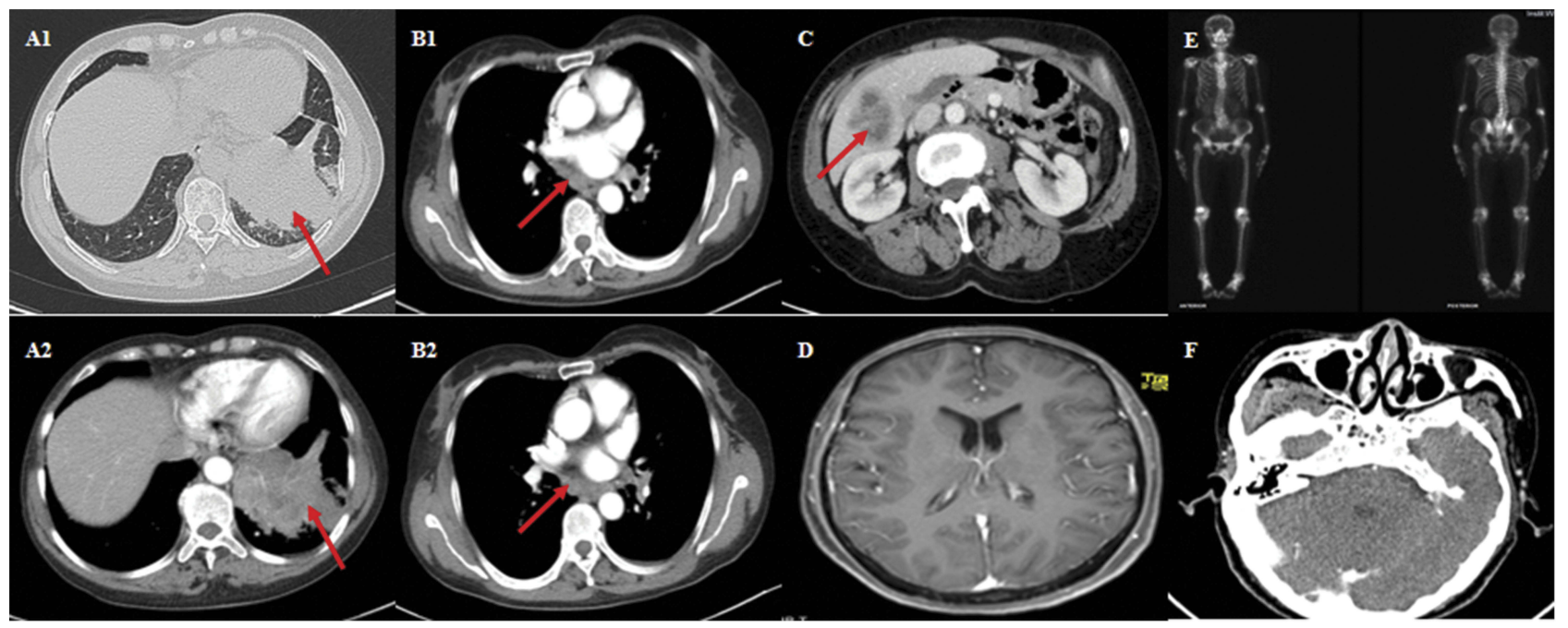

Figure I The images of enhanced CT of chest, whole abdomen, brain and nasopharyngeal, and the whole body bone scan at the first time point. (AI, A2, B I, B2) A bulky soft tissue mass in the left lower lobe with lobulated, burr sign. Furthermore, the left lung door and mediastinal lymph nodes showed enlargement. (C) A kind of circular low density shadow in the liver. (D-F) Some focal ischemia in the brain, deputy nose and hypertrophy of bilateral inferior turbinate, and no bone metastasis (July 26, 2018). 


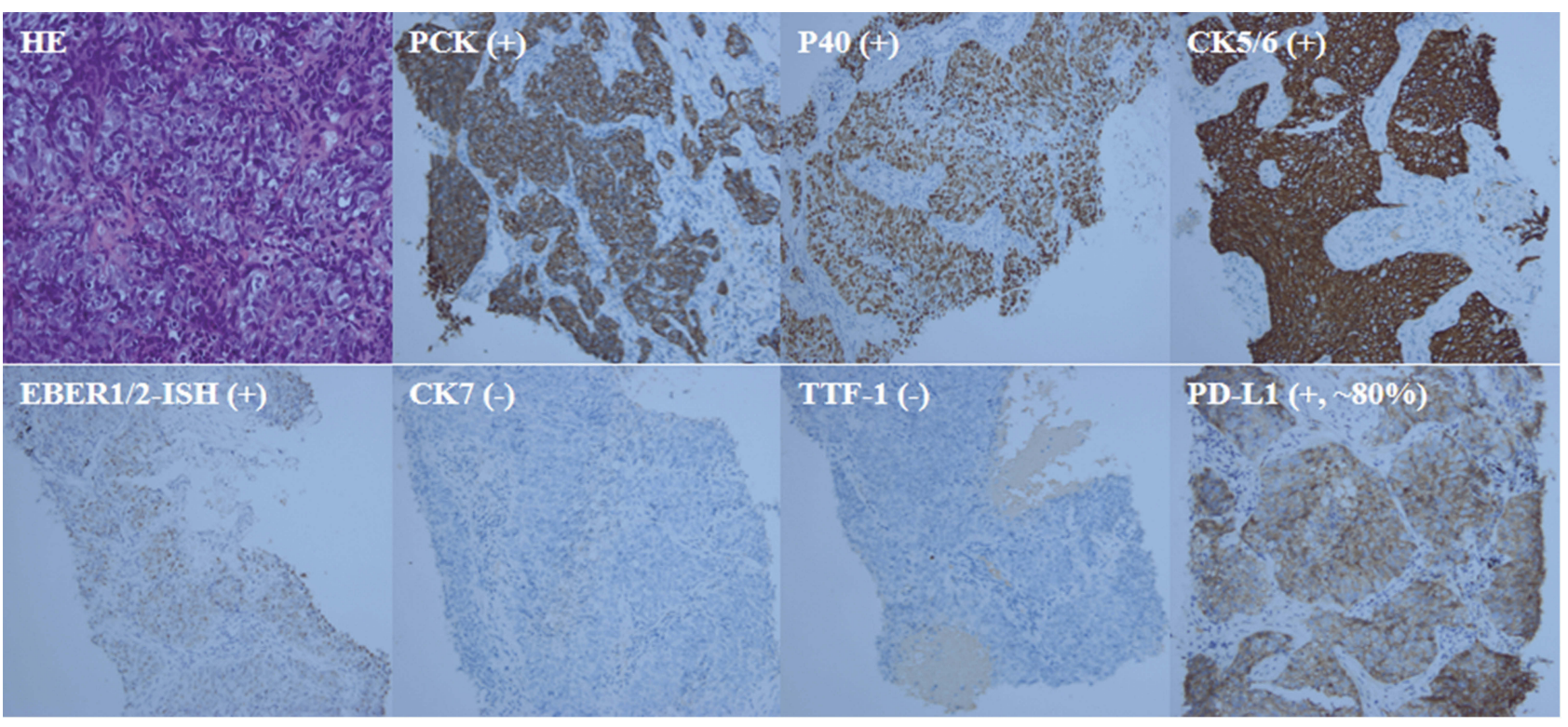

Figure 2 The IHC staining of percutaneous lung biopsy with different antibodies. IHC staining demonstrated PCK (+), P40 (+), CK5/6 (+), EBERI/2-ISH (+), CK7 (-), TTF-I $(-)$, and PD-LI (+, positive proportion about $80 \%)$. Original magnification, $\times 400$ in $\mathrm{HE}, \times 200$ in others.

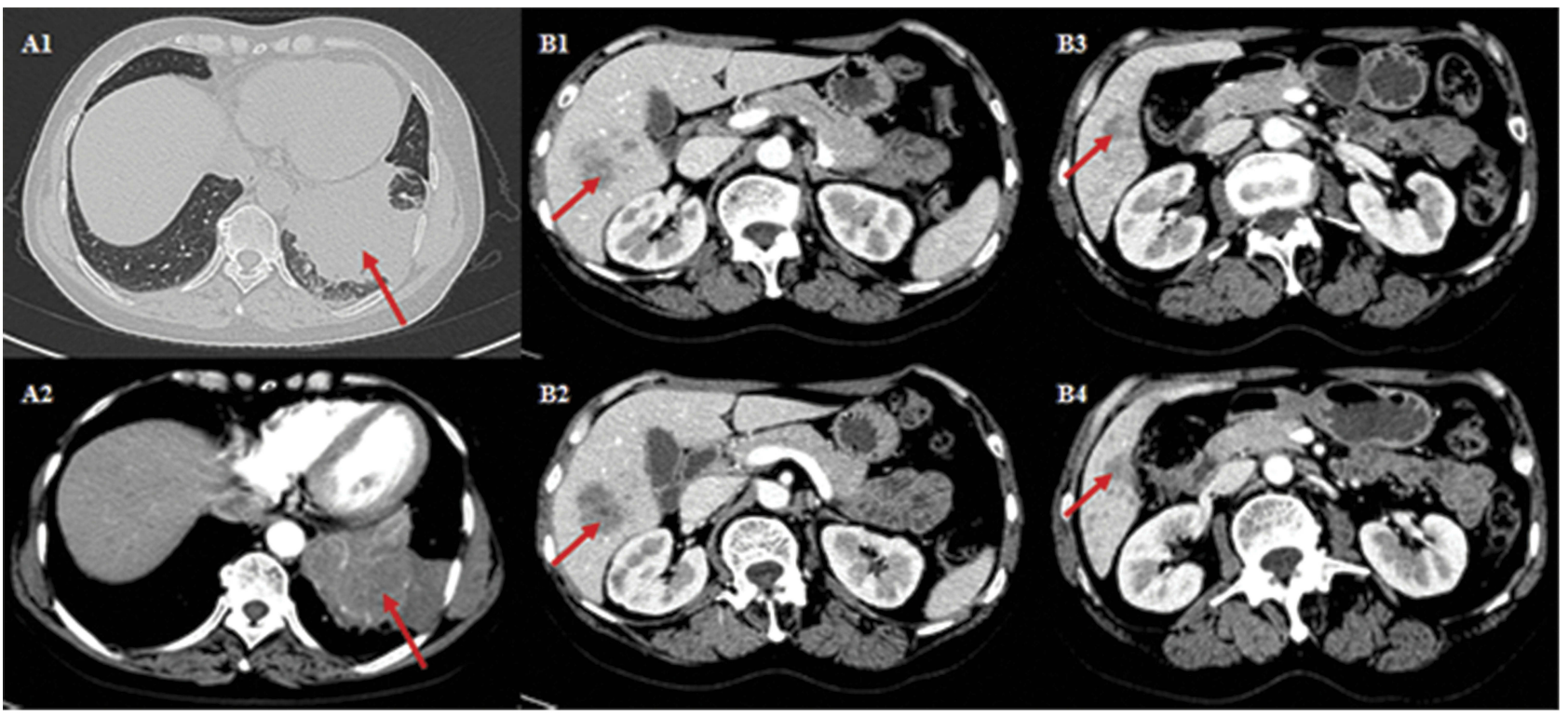

Figure 3 The images of enhanced CT of chest and whole abdomen after two cycles of chemotherapy. (AI, A2) A bulky soft tissue mass in the left lower lobe, which was larger than before. (B I-B4) Multiple classes of circular low density shadows in the liver, and the lesions of liver had become broader than before (October 29, 20I8).

PD-L1, we decided to stop the chemotherapy and began to use nivolumab (Opdivo; Bristol-Myers Squibb, New York, NY). Subsequently, the patient was administered nivolumab $3 \mathrm{mg} / \mathrm{kg}$ every 14 days. After two cycles, we evaluated the curative effect again, and to our surprise the size of the mass had reduced significantly (Figures 5A1 and A2, evaluation result was PR), and there was no metastasis in the brain (Figure 5B). After five cycles of nivolumab, the size of the tumor and the lesions of the liver became even smaller (Figure 6, evaluation result was PR). At the same time, we performed the blood tests again and found that the values of CYFRA21-1 and NSE dramatically decreased compared to before, especially the CYFRA21-1 (Figure 7). So far, this patient has finished six cycles of treatment of nivolumab and there have been no obvious side-effects during the treatment process except an occasional mild cough. Meantime, the hormone level of the thyroid and cortisol have remained roughly normal. 


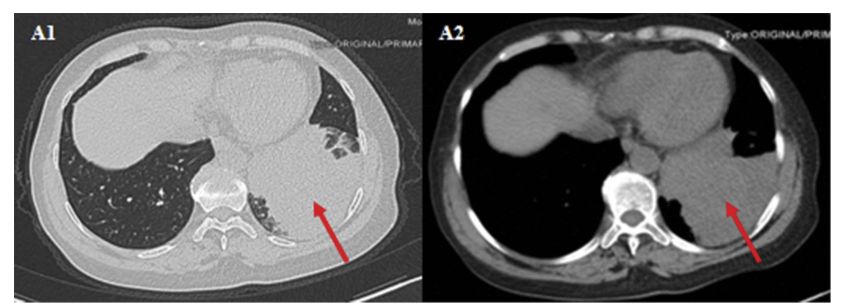

Figure 4 The images of chest CT after four cycles of chemotherapy. (AI, A2) The mass became bigger than before (December 25, 2018). The arrow pointed to the mass, which was bigger than before.

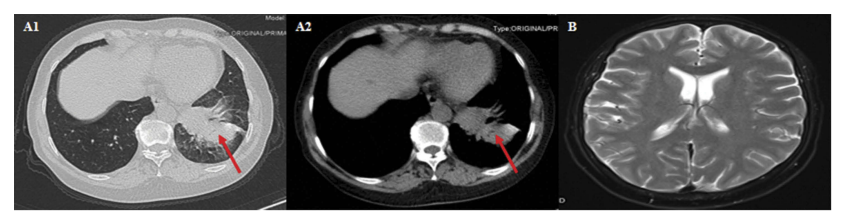

Figure 5 The images of chest and brain CT after two cycles of immunotherapy. (Al, A2) The mass the size of the mass has reduced significantly. (B) No metastasis in the brain (February 2I, 20I9). The arrow pointed to the mass, which reduced after 2 cycles of immunotherapy.

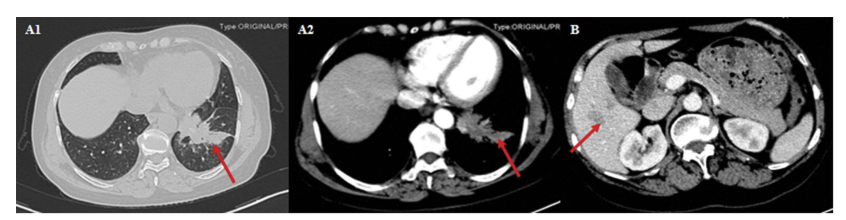

Figure 6 The images of enhanced CT of the chest and whole abdomen after five cycles of immunotherapy. (AI, A2) The size of the mass has reduced significantly. (B) No metastasis in the brain (May 5, 2019). The arrow pointed to the mass, which became more smaller after 5 cycles of immunotherapy.

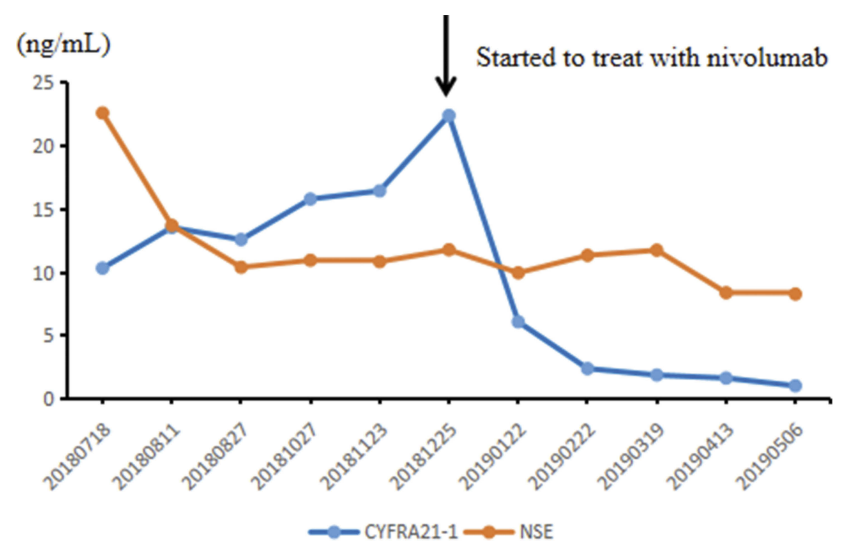

Figure 7 The curves of CYFRA2I-I and NSE during the treatment. The values of CYFRA2I-I and NSE dramatically decreased compared to before immunotherapy, especially the CYFRA2I-I.

\section{Discussion}

We have described the diagnosis and treatment process of a patient with stage IVA primary pulmonary LELC in the present case. A case report of three patients with LELC treated with
PD-1 antibody was reported recently. Among these cases, two of them with nivolumab did not result in an objective response, but with rapidly progressing disease. ${ }^{10,11}$ Only one patient experienced a partial response to atezolizumab for six cycles that lasted 4 months. ${ }^{12}$ To the best of our knowledge, this case is the first report of primary pulmonary LELC responding favorably to nivolumab. Our patient experienced a partial response to nivolumab for six cycles that lasted 5 months. During this time, her tumor burden had decreased significantly.

Pulmonary LELC is a rare malignancy, estimated to represent $0.15 \%$ to $3.6 \%$ of all lung malignancies, and is usually associated with EBV infection, which, at one point, was even thought to be the main etiology of LELC. ${ }^{12,13}$ LELC is more commonly found in Asia, and most cases have been reported in Southeast Asia, specifically Hong Kong, Taiwan, and Guangdong. ${ }^{7}$ Our patient came from southwest of China, and IHC showed a positive EBER1/2-ISH. Furthermore, this patient had the same clinical and radiographic features with literature reported before, such as being a middle-aged women with no-smoking history, the large size of tumor at the central lesions, and the values of serum tumor markers of CYFRA211 and NSE were usually high. ${ }^{14}$

Immune checkpoint inhibitors, targeting cytotoxic T-lymphocyte antigen-4 (CTLA-4) and programmed death-1 (PD-1), are a recent addition to the treatment arsenal against different types of advanced cancers, including NSCLC. ${ }^{10,15}$ Nivolumab and pembrolizumab, as anti-PD-1 antibodies, have shown survival benefits over standard second-line chemotherapy for advanced NSCLC, and are approved for treatment of unresectable NSCLC after progression on platinum containing chemotherapy, or appropriate molecular-targeted therapy in patients with EGFR-sensitizing mutations or ALK translocations. ${ }^{16,17}$ The mechanism of immune checkpoint blockade in pulmonary LELC remains unclear, but activity of immune checkpoint inhibitors observed in other virus-associated cancers warrants further evaluation of this new class of cancer therapeutics in patients with pulmonary LELC. El-Khoueiry et al ${ }^{18}$ indicated that the objective response rate (ORR) in patients with virus-associated hepatocellular carcinoma (HCC) treated with nivolumab was $32 \%$, which was higher than in uninfected patients with HCC (ORR, 15\%). Additionally, the use of pembrolizumab for the treatment of recurrent or metastatic squamous cell carcinoma led to a higher ORR in patients with human papillomavirus (HPV)-positive tumor (ORR=25\%), compared to those with HPV-negative $(\mathrm{ORR}=14 \%){ }^{19}$ Similarly, in patients with Merkel-cell carcinoma, a skin cancer linked to the Merkelcell polyomavirus, the ORR was $56 \%$ after treatment with 
pembrolizumab. ${ }^{20}$ Several studies reported that tumors expressing PD-L1 are more likely to respond to PD-1/PD-L1 blockade. ${ }^{17,21-23}$ Our patient had high expression of PD-L1 with a positive rate about $80 \%$, consistent with previously research. However, PD-L1 expression on tumor cells was predictive of treatment outcomes, including ORR and OS in patients with non-squamous NSCLC, but not in those with squamous NSCLC in two phase III trials of nivolumab in NSCLC. ${ }^{10,16,24}$ In summary, the findings below provide a rationale to treat patients with pulmonary LELC with immune checkpoint therapy.

\section{Conclusion}

We have reported the case of an Asian patient with EBV-positive primary pulmonary LELC. Our patient responded well to immunotherapy with nivolumab. Although the effectiveness of checkpoint inhibition in pulmonary LELC will be difficult to assess owing to the low incidence rate, the findings from our present case report indicate that EBV-positive pulmonary LELC with high expression of PD-L1 may derive benefit from a PD-1/ PD-L1 blockade. However, specific trials of immunotherapy for rare lung cancer cases are still warranted.

\section{Consent Statement}

Written and informed consent has been provided by the patient to have the case details and any accompanying images published.

The director of West China Hospital Weimin Li clarified: The manuscript of "Primary Pulmonary Lymphoepitheliomalike Carcinoma Response Favorably to Nivolumab: A Case Report" by Dr. Qiu et al has been informed for publication. All case details and any accompanying images have been checked and approved.

\section{Funding}

This work was supported by the National Science Foundation of China $(81700095,81870034)$.

\section{Disclosure}

The authors report no conflicts of interest in this work.

\section{References}

1. Lin Z, Situ D, Chang X, et al. Surgical treatment for primary pulmonary lymphoepithelioma-like carcinoma. Interact Cardiovasc Thorac Surg. 2016;23:41-46. doi:10.1093/icvts/ivw064

2. Begin LR, Eskandari J, Joncas J, Panasci L. Epstein-Barr virus related lymphoepithelioma-like carcinoma of lung. $J$ Surg Oncol. 1987;36:280-283. doi:10.1002/jso.2930360413
3. Ho JC, Wong MP, Lam WK. Lymphoepithelioma-like carcinoma of the lung. Respirology. 2006;11:539-545. doi:10.1111/j.1440-1843.20 06.00910.x

4. Chang YL, Wu CT, Shih JY, Lee YC. New aspects in clinicopathologic and oncogene studies of 23 pulmonary lymphoepithelioma-like carcinomas. Am J Surg Pathol. 2002;26:715-723. doi:10.1097/0000 0478-200206000-00004

5. Lin CY, Chen YJ, Hsieh MH, Wang CW, Fang YF. Advanced primary pulmonary lymphoepithelioma-like carcinoma: clinical manifestations, treatment, and outcome. J Thorac Dis. 2017;9:123-128. doi:10.21037/jtd.2017.01.25

6. Liang Y, Wang L, Zhu Y, et al. Primary pulmonary lymphoepithelioma-like carcinoma: fifty-two patients with long-term follow-up. Cancer. 2012;118:4748-4758. doi:10.1002/cncr.27452

7. Huang CJ, Feng AC, Fang YF, et al. Multimodality treatment and long-term follow-up of the primary pulmonary lymphoepitheliomalike carcinoma. Clin Lung Cancer. 2012;13:359-362. doi:10.1016/j. cllc.2012.01.002

8. Han AJ, Xiong M, Gu YY, Lin SX, Xiong M. Lymphoepitheliomalike carcinoma of the lung with a better prognosis. A clinicopathologic study of 32 cases. Am J Clin Pathol. 2001;115:841-850. doi:10.1309/BUAN-BGFW-69U9-C3H8

9. Chan AT, Teo PM, Lam KC, et al. Multimodality treatment of primary lymphoepithelioma-like carcinoma of the lung. Cancer. 1998;83:925929. doi:10.1002/(sici)1097-0142(19980901)83:5<925::aid-cncr18>3.0. co;2-x

10. Kim C, Rajan A, DeBrito PA, Giaccone G. Metastatic lymphoepithelioma-like carcinoma of the lung treated with nivolumab: a case report and focused review of literature. Transl Lung Cancer Res. 2016;5:720-726. doi:10.21037/tlcr.2016.11.06

11. Darrason M, Martin A, Soussan M, et al. Immunotherapy for LELC: case report and a focused review. Clin Lung Cancer. 2019;20:e393e401. doi:10.1016/j.cllc.2018.12.008

12. Narayanan A, Knollmann FD, Walby JAS, Lim S, Gandara DR, Riess JW. EBV-positive primary pulmonary lymphoepithelioma-like carcinoma response to PD-L1 blockade. Clin Lung Cancer. 2019;20:e238e241. doi:10.1016/j.cllc.2018.12.015

13. Ngan RK, Yip TT, Cheng WW, et al. Clinical role of circulating Epstein-Barr virus DNA as a tumor marker in lymphoepitheliomalike carcinoma of the lung. Ann N Y Acad Sci. 2004;1022:263-270. doi:10.1196/annals.1318.041

14. Qin Y, Gao G, Xie X, et al. Clinical features and prognosis of pulmonary lymphoepithelioma-like carcinoma: summary of eightyfive cases. Clin Lung Cancer. 2019;20:e329-e337. doi:10.1016/j. cllc.2018.12.014

15. Pardoll DM. The blockade of immune checkpoints in cancer immunotherapy. Nat Rev Cancer. 2012;12:252-264. doi:10.1038/nrc3239

16. Borghaei H, Paz-Ares L, Horn L, et al. Nivolumab versus docetaxel in advanced nonsquamous non-small-cell lung cancer. $N$ Engl J Med. 2015;373:1627-1639. doi:10.1056/NEJMoa1507643

17. Herbst RS, Baas P, Kim DW, et al. Pembrolizumab versus docetaxel for previously treated, PD-L1-positive, advanced nonsmall-cell lung cancer (KEYNOTE-010): a randomised controlled trial. Lancet. 2016;387:1540-1550. doi:10.1016/S0140-6736(15) 01281-7

18. Ben-Josef E, Guthrie KA, El-Khoueiry AB, et al. SWOG S0809: a phase ii intergroup trial of adjuvant capecitabine and gemcitabine followed by radiotherapy and concurrent capecitabine in extrahepatic cholangiocarcinoma and gallbladder carcinoma. J Clin Oncol. 2015;33:2617-2622. doi:10.1200/JCO.2014.60.2219

19. Kueh HY, Yui MA, Ng KK, et al. Asynchronous combinatorial action of four regulatory factors activates $\mathrm{Bcl11} \mathrm{b}$ for $\mathrm{T}$ cell commitment. Nat Immunol. 2016;17:956-965. doi:10.1038/ni.3514

20. Nghiem PT, Bhatia S, Lipson EJ, et al. PD-1 blockade with pembrolizumab in advanced merkel-cell carcinoma. $N$ Engl $J$ Med. 2016;374:2542-2552. doi:10.1056/NEJMoa1603702 
21. Herbst RS, Soria JC, Kowanetz M, et al. Predictive correlates of response to the anti-PD-L1 antibody MPDL3280A in cancer patients. Nature. 2014;515:563-567. doi:10.1038/nature14011

22. Garon EB, Rizvi NA, Hui R, et al. Pembrolizumab for the treatment of non-small-cell lung cancer. $N$ Engl J Med. 2015;372:2018-2028. doi:10.1056/NEJMoa1501824
23. McLaughlin J, Han G, Schalper KA, et al. Quantitative assessment of the heterogeneity of PD-L1 expression in non-small-cell lung cancer. JAMA Oncol. 2016;2:46-54. doi:10.1001/jamaoncol.2015.3638

24. Brahmer J, Reckamp KL, Baas P, et al. Nivolumab versus docetaxel in advanced squamous-cell non-small-cell lung cancer. $N$ Engl $J$ Med. 2015;373:123-135. doi:10.1056/NEJMoa1504627

\section{Publish your work in this journal}

OncoTargets and Therapy is an international, peer-reviewed, open access journal focusing on the pathological basis of all cancers, potential targets for therapy and treatment protocols employed to improve the management of cancer patients. The journal also focuses on the impact of management programs and new therapeutic

Submit your manuscript here: https://www.dovepress.com/oncotargets-and-therapy-journal agents and protocols on patient perspectives such as quality of life, adherence and satisfaction. The manuscript management system is completely online and includes a very quick and fair peer-review system, which is all easy to use. Visit http://www.dovepress.com/ testimonials.php to read real quotes from published authors. 